\title{
Relativistic BCS-BEC Crossover at Quark Level
}

Lianyi He, Shijun Mao, and Pengfei Zhuang

Physics Department, Tsinghua University, Beijing 100084, China

\begin{abstract}
The non-relativistic $G_{0} G$ formalism of BCS-BEC crossover at finite temperature is extended to relativistic fermion systems. The theory recovers the BCS mean field approximation at zero temperature and the non-relativistic results in a proper limit. For massive fermions, when the coupling strength increases, there exist two crossovers from the weak coupling BCS superfluid to the non-relativistic BEC state and then to the relativistic BEC state. For color superconductivity at moderate baryon density, the matter is in the BCS-BEC crossover region, and the behavior of the pseudogap is quite similar to that found in high temperature superconductors.
\end{abstract}

\section{Acknowledgements}

The work is supported by the NSFC Grant 10735040 and the 973-project 2006CB921404 and 2007CB815000.

\section{References}

1. A.J.Leggett, Modern trends in the theory of condensed matter, Springer-Verlag, Berlin (1980)

2. P.Nozieres and S.Schmitt-Rink, J.Low.Temp.Phys. 59, 195 (1985)

3. Q.Chen, J.Stajic, S.Tan and K.Levin, Phys.Rept. 412, 1 (2005)

4. Y. Nishida and H. Abuki, Phys.Rev. D72, 096004, (2005)

5. J.Deng, A.Schmitt and Q.Wang, Phys.Rev. D78, 034014 (2008)

6. L.He and P.Zhuang, Phys.Rev. D76, 056003 (2007); Phys.Rev. D75, 096004 (2007); Phys.Rev. D75, 096003 (2007) 


\section{Relativistic BCS-BEC Crossover at Quark Level Pengfei Zhuang \\ Physics Department, Tsinghua University, Beijing 100084}
1) Motivation
2) Mean Field Theory at $T=0$
3) Fluctuations at $T \neq 0$
4) Application to QCD:
Color Superconductivity and Pion Superfluid
5) Conclusions

1) Motivation 
$B C S-B E C$

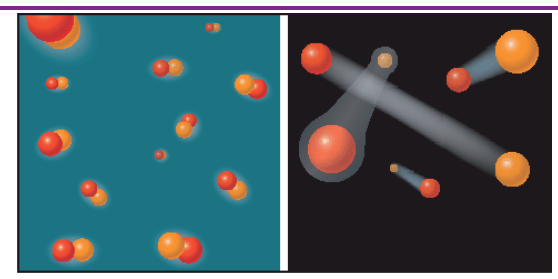

BEC of molecules

BCS (Barden, Cooper and Schrieffer, 1957): normal superconductivity weak coupling, large pair size, $\boldsymbol{k}$-space pairing, overlaping Cooper pairs BEC (Bose-Einstein-Condensation, 1924/1925):

strong coupling, small pair size, $r$-space pairing, ideal gas of bosons, first realization in dilute atomic gas with bosons in 1995.

BCS-BEC crossover (Eagles, Leggett, 1969, 1980):

$B C S$ wave function at $T=0$ can be generalized to arbitrary attraction: a smooth crossover from $B C S$ to $B E C$ !

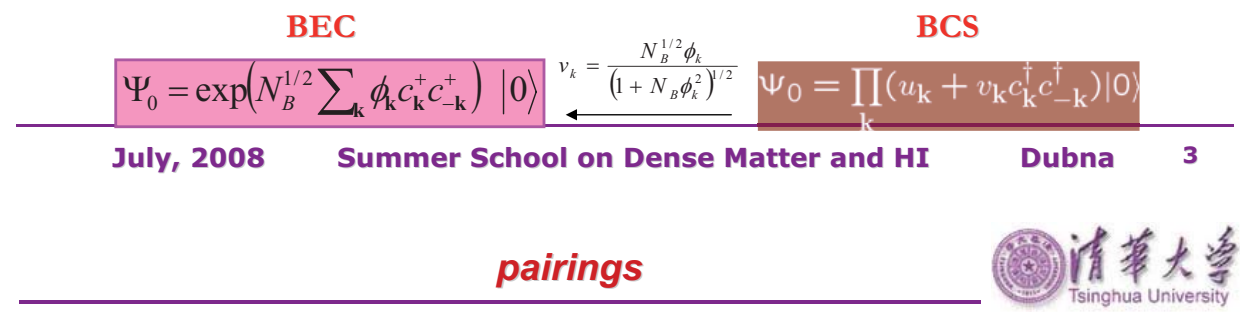

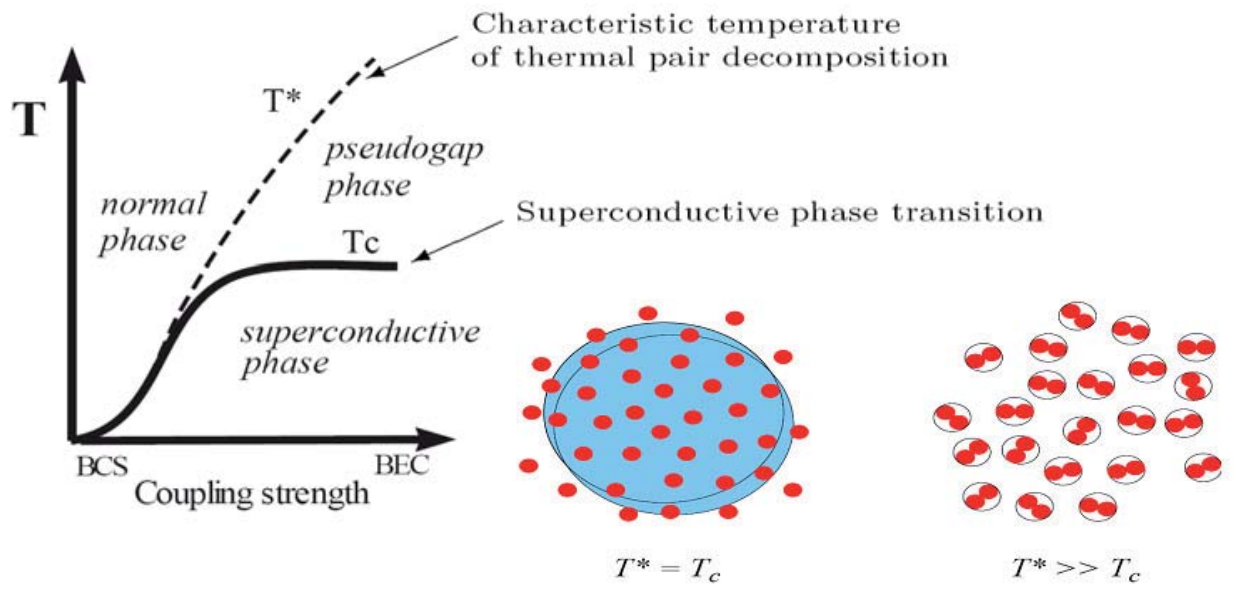

in BCS, $T_{c}$ is determined by thermal excitation of fermions, in BEC, $T_{c}$ is controlled by thermal excitation of collective modes 
$B C S-B E C$ in QCD

in order to study the question of 'vacuum', we must turn to a different direction: we should investigate some 'bulk' phenomena by distributing high energy over a relatively large volume.

T. D. Lee, Rev. Mod. Phys. 47, 267(1975)
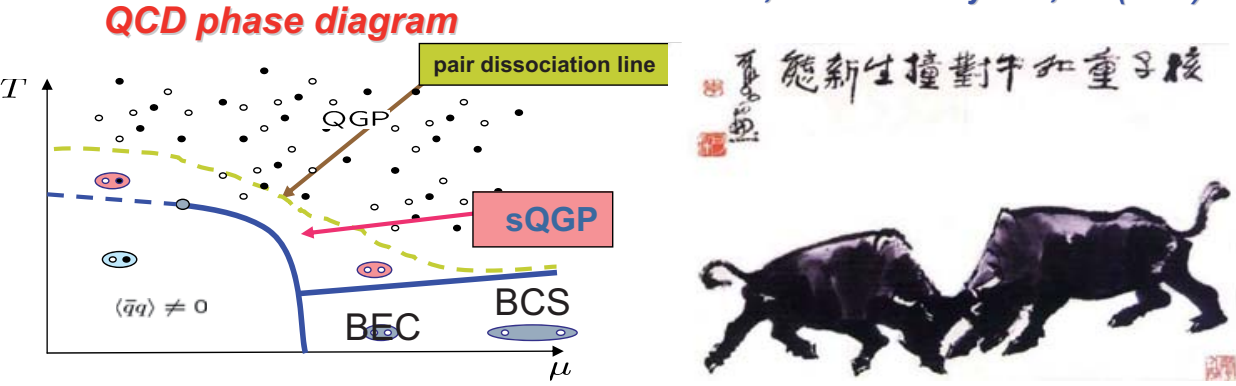

rich QCD phase structure at high density, natural attractive interaction in QCD, possible BCS-BEC crossover?

new phenomena in BCS-BEC crossover of QCD:

relativistic systems, anti-fermion contribution, rich inner structure (color, flavor), medium dependent mass, .......

July, 2008 Summer School on Dense Matter and HI Dubna 5

theory of BCS-BEC crossover

*) Leggett mean field theory (Leggett, 1980)

*)NSR scheme (Nozieres and Schmitt-Rink, 1985)

extension of of BCS-BEC crossover theory at $T=0$ to $T \neq 0$ (above $T_{c}$ ) Nishida and Abuki $(2006,2007)$

extension of non-relativistic NSR theory to relativistic systems, BCS-NBEC-RBEC crossover

$$
\Omega_{f l}=\int \frac{d^{4} q}{(2 \pi)^{4}} \ln \left[\frac{1}{G}-\chi(q)\right], \quad \chi=\bigcirc \square G_{0} G_{0}
$$

*) $G_{0}$ G scheme (Chen, Levin et al., 1998, 2000, 2005)

$$
\text { asymmetric pair susceptibility } \chi=\bigodot \square G_{0} G
$$

extension of non-relativistic $G_{0} G$ scheme to relativistic systems (He, Zhuang, 2006, 2007)

*) Bose-fermion model (Friedderg, Lee, 1989, 1990)

extension to relativistic systems (Deng, Wang, 2007)

Kitazawa, Rischke, Shovkovy, 2007, NJL+phase diagram Brauner, 2008, collective excitations 
2) Leggett Mean Field Theory at $T=0$

A.J.Leggett, in Modern trends in the theory of condensed matter, Springer-Verlag (1980)

July, 2008 Summer School on Dense Matter and HI

Dubna 7

non-relativistic mean field theory

fermion number:

$n$

Fermi momentum: $\quad k_{F}, n=k_{F}^{3} / 3 p^{2}$

Fermi energy:

$e_{F}=k_{F}^{2} / 2 m$

$L=\psi_{\sigma}^{+}\left(i \partial_{t}-\frac{\nabla^{2}}{2 m}-\sigma_{3} h\right) \psi_{\sigma}+g \psi_{\uparrow}^{+} \psi_{\downarrow}^{+} \psi_{\downarrow} \psi_{\uparrow}$

order parameter $\quad \Delta=2 g\left\langle\psi_{\downarrow}^{+} \psi_{\downarrow}\right\rangle=2 g\left\langle\psi_{\uparrow}^{+} \psi_{\uparrow}\right\rangle$

thermodynamics in mean field approximation

$$
\Omega=\frac{\Delta^{2}}{g}-\int \frac{d^{3} \vec{k}}{(2 \pi)^{3}}\left[E_{k}-\xi_{k}-\frac{\Delta^{2}}{4 \varepsilon_{k}}\right]
$$

quasi-particle energy $E_{k}=\sqrt{x_{k}^{2}+\mathrm{D}^{2}}, \quad x_{k}=e_{k}-m, \quad e_{k}=k^{2} / 2 m$

renormalization to avoid the integration divergence

$$
\begin{aligned}
& g \rightarrow a_{s}, \quad \frac{m}{4 \pi a_{s}}=\frac{1}{g}+\int \frac{d^{3} k}{(2 \pi)^{3}} \frac{1}{2 \varepsilon_{k}} \\
& a_{s}<0 \text { for attractive coupling, } a_{s}>0 \text { for repulsive coupling }
\end{aligned}
$$


gap equation $\frac{\partial \Omega}{\partial \Delta}=0$

number conservation $n=-\frac{\partial \Omega}{\partial \mu}$

$$
\Rightarrow \Delta(n), \quad \mu(n)
$$

$$
\left\{\begin{aligned}
-\frac{m}{4 \pi a_{s}} & =\int \frac{d^{3} k}{(2 \pi)^{3}}\left(\frac{1}{2 E_{k}}-\frac{1}{2 \varepsilon_{k}}\right) \\
n & =\int \frac{d^{3} k}{(2 \pi)^{3}}\left(1-\frac{\xi_{k}}{E_{k}}\right)
\end{aligned}\right.
$$

universality

$$
\begin{aligned}
& \hat{\mu}=\mu / \varepsilon_{F}, \quad \tilde{\Delta}=\Delta / \varepsilon_{F}, \quad \eta=\frac{1}{k_{F} a_{s}} \quad \text { effective coupling } \\
& \left\{\begin{array}{l}
\eta \frac{\eta}{2}=\int_{0}^{\infty} d x x^{2}\left(\frac{1}{\sqrt{\left(x^{2}-\hat{\mu}\right)^{2}+\tilde{\Delta}^{2}}}-\frac{1}{x^{2}}\right) \\
\frac{2}{3}=\int_{0}^{\infty} d x x^{2}\left(1-\frac{x^{2}-\hat{\mu}}{\sqrt{\left(x^{2}-\tilde{\mu}\right)^{2}+\tilde{\Delta}^{2}}}\right)
\end{array}\right.
\end{aligned}
$$

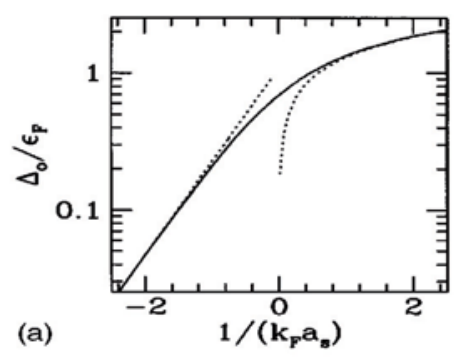

$$
\begin{aligned}
& \text { BCS limit } \\
& \eta \rightarrow-\infty, \quad \tilde{\Delta}=\frac{8}{e^{2}} e^{2 \eta / \pi}, \quad \hat{\mu}=1 \\
& \text { BEC limit } \\
& \eta \rightarrow \infty, \quad \tilde{\Delta}=\sqrt{\frac{16 \eta}{3 \pi}}, \quad \hat{\mu}=-\eta^{2} \\
& \mu=-\frac{\varepsilon_{b}}{2}, \quad \varepsilon_{b}=\frac{1}{m a_{s}^{2}} \\
& n(p)=\frac{1}{e^{(\varepsilon-\mu) / T}-1} \quad \Rightarrow \quad \mu \leq 0
\end{aligned}
$$

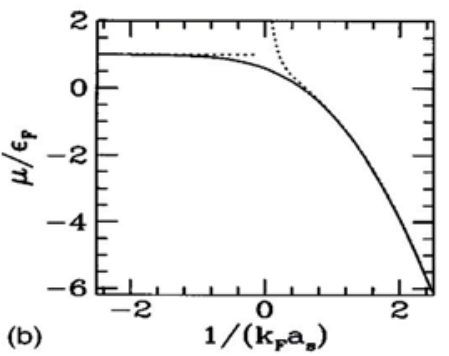

\section{BCS-BEC crossover}

$$
\begin{aligned}
& \eta<0 \rightarrow \eta>0, \\
& \text { small } \Delta \rightarrow \text { large } \Delta, \\
& \mu>0 \rightarrow \mu<0
\end{aligned}
$$




$$
\begin{aligned}
& E_{k}^{ \pm}=\sqrt{\left(x_{k}^{ \pm}\right)^{2}+\mathrm{D}^{2}}, \quad x_{k}^{ \pm}=\sqrt{k^{2}+m^{2}} \pm m \\
& m-m \text { plays the role of non-relativistic chemical potential }
\end{aligned}
$$

BCS-BEC crossover around

$$
m-m=0, \quad m=m
$$

pair binding energy

$$
\begin{aligned}
& e_{b}<2 m \\
& m-m>-\frac{e_{b}}{2} \mathrm{i}-m, \quad m \mathrm{i} 0
\end{aligned}
$$

at $m \AA 0$ fermion and anti-fermion degenerate, relativistic effect

\begin{tabular}{lcccc}
$\begin{array}{l}\text { RBEC } \\
\text { limit }\end{array}$ & $\begin{array}{l}\text { RBEC-NBEC } \\
\text { crossover }\end{array}$ & NBEC & $\begin{array}{l}\text { NBEC-BCS } \\
\text { crossover }\end{array}$ & $\begin{array}{l}\text { BCS } \\
\text { limit }\end{array}$ \\
\hline 0 & & $m$ & $m$
\end{tabular}

July, 2008 Summer School on Dense Matter and HI Dubna 11

relativistic mean field theory

extremely high $T$ and high density: $p Q C D$

finite $T$ (zero density): lattice QCD

moderate $T$ and density: models like 4-fermion interaction (NJL)

$$
\begin{aligned}
& L=\bar{\psi}\left(i \gamma^{\mu} \partial_{\mu}-m\right) \psi+\frac{g}{4}\left(\bar{\psi} i \gamma_{5} C \bar{\psi}^{T}\right)\left(\psi^{T} i C \gamma_{5} \psi\right) \\
& C=i \gamma_{0} \gamma_{2} \text { charge conjunction matrix }
\end{aligned}
$$

order parameter $\Delta=\frac{g}{2}\left\langle\psi^{T} i C \gamma_{5} \psi\right\rangle$

mean field thermodynamic potential

$$
\begin{gathered}
\Omega=\frac{\Delta^{2}}{g}-\int \frac{d^{3} \vec{k}}{(2 \pi)^{3}}\left[E_{k}^{+}+E_{k}^{-}-\xi_{k}^{+}-\xi_{k}^{-}\right] \\
\text {antifermion }
\end{gathered} \overbrace{\text { fermion }}
$$

the most important thermodynamic contribution from the uncondensed pairs is from the Goldstone modes, $\varepsilon \square T^{4}$, at $T=0$, the fluctuation contribution disappears, and MF is a good approximation at $T=0$. 


$$
\left\{\begin{array}{c}
\frac{1}{g}=\int \frac{d^{3} k}{(2 \pi)^{3}}\left(\frac{1}{2 E_{k}^{-}}-\frac{1}{2 E_{k}^{+}}\right) \\
n=\int \frac{d^{3} k}{(2 \pi)^{3}}\left[\left(1-\frac{\xi_{k}^{-}}{E_{k}^{-}}\right)-\left(1-\frac{\xi_{k}^{+}}{E_{k}^{+}}\right)\right]
\end{array}\right.
$$

renormalization to avoid the integration divergence

$$
-\frac{1}{U}=\frac{1}{g}-\frac{1}{2} \int \frac{d^{3} k}{(2 \pi)^{3}}\left(\frac{1}{\varepsilon_{k}-m}+\frac{1}{\varepsilon_{k}+m}\right), \quad U=\frac{4 \pi a_{s}}{m}
$$

the ultraviolet divergence can not be completely removed, and a momentum cutoff $\Lambda$ still exists in the theory.

broken universality:

$$
\left\{\begin{array}{c}
-\frac{\pi}{2} \eta=\int_{0}^{z} d x x^{2}\left[\left(\frac{1}{E_{x}^{-}}-\frac{1}{\varepsilon_{x}-2 \zeta^{-2}}\right)+\left(\frac{1}{E_{x}^{+}}-\frac{1}{\varepsilon_{x}+2 \zeta^{-2}}\right)\right] \\
\frac{2}{3}=\int_{0}^{z} d x x^{2}\left[\left(1-\frac{\xi_{x}^{-}}{E_{x}^{-}}\right)-\left(1-\frac{\xi_{x}^{+}}{E_{x}^{+}}\right)\right]
\end{array}\right.
$$

$\eta=\frac{1}{k_{F} a_{s}}, \quad \zeta=\frac{k_{F}}{m} \quad$ explicit density dependence !

July, 2008 Summer School on Dense Matter and HI Dubna 13

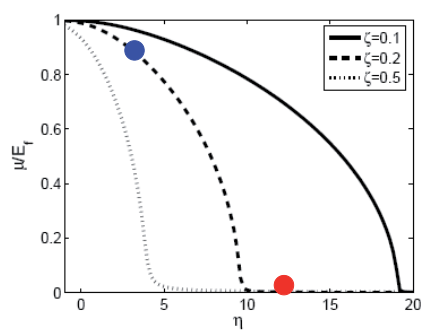

\section{non-relativistic limit}
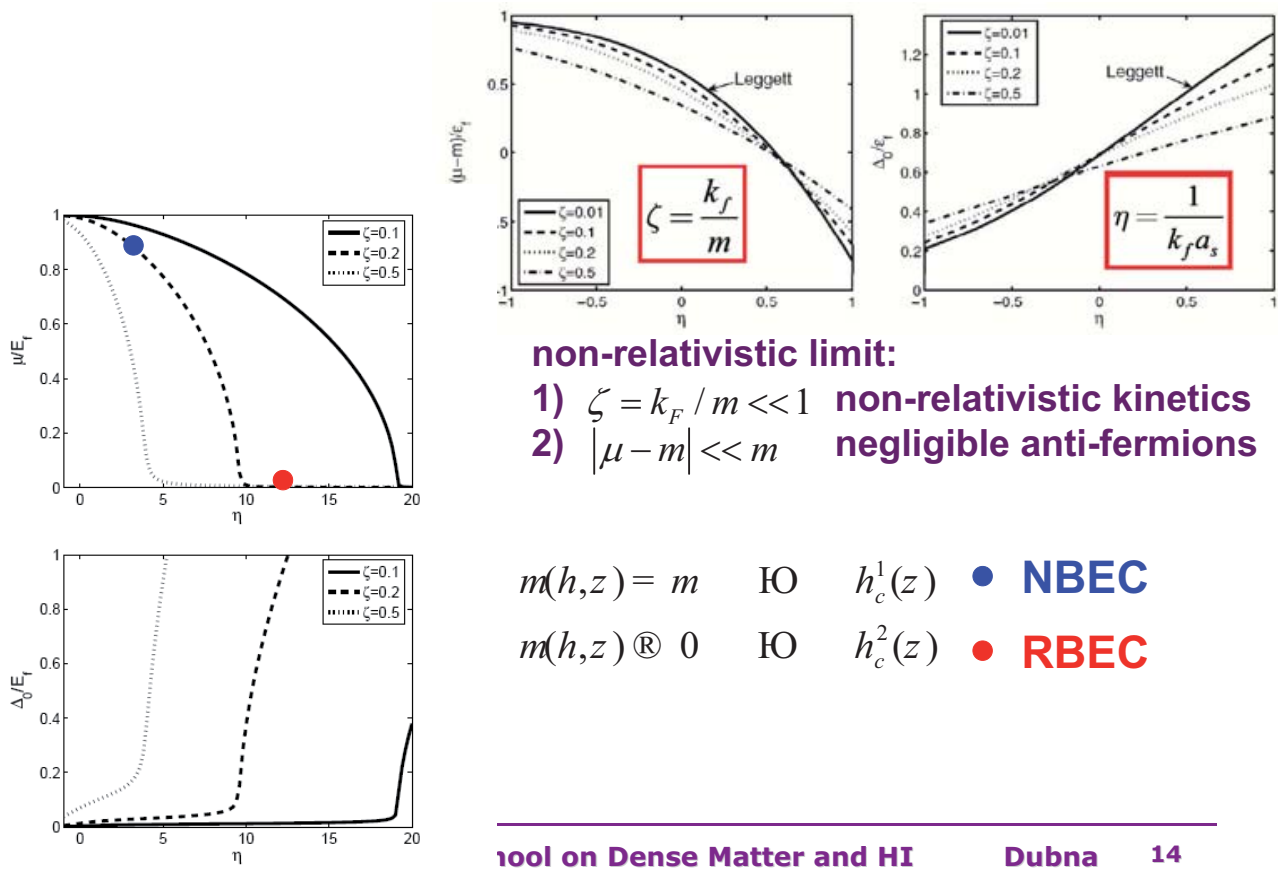

non-relativistic limit:

1) $\zeta=k_{F} / m<<1$ non-relativistic kinetics

2) $|\mu-m|<<m$ negligible anti-fermions

$$
\begin{aligned}
& m(h, z)=m \quad \text { Ю } \quad h_{c}^{1}(z) \bullet \text { NBEC } \\
& m(h, z) \circledR 0 \quad \text { Ю } h_{c}^{2}(z) \bullet \text { RBEC }
\end{aligned}
$$


In non-relativistic case, only one dimensionless variable, $\eta=1 / k_{F} a_{s}$, changing the density of the system can not induce a BCS-BEC crossover.

However, in relativistic case, the extra density dependence $z=k_{F} / m$ may induce a BCS-BEC crossover.

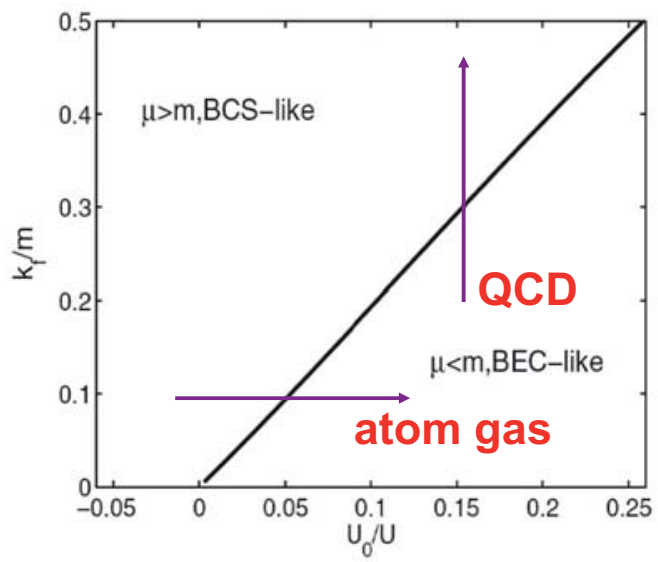

3) Fluctuations at $T \neq 0$ 
- the Landau mean field theory is a good approximation only at $\mathrm{T}=0$ where there is no thermal excitation, one has to go beyond the mean field at finite temperature.

- an urgent question in relativistic heavy ion collisions

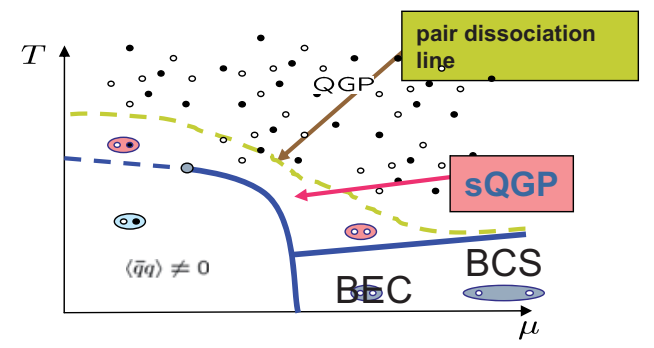

to understand the SQGP phase with possible bound states of quarks and gluons, one has to go beyond the mean field!

- going beyond mean field self-consistently is very difficult NSR Theory ( $G_{0} G_{0}$ Scheme) above $T_{c}$ : Nishida and Abuki (2005), Bose-Fermi Model: Deng and Wang (2006), $G_{0} G$ Scheme below $T_{c}$ : He and Zhuang (2007), ...... July, 2008 Summer School on Dense Matter and HI Dubna 17 mean field fermion propagator

gap and number equations at finite $T$

$$
L=\bar{\psi}\left(i \gamma^{\mu} \partial_{\mu}-m\right) \psi+\frac{g}{4}\left(\bar{\psi} i \gamma_{5} C \bar{\psi}^{T}\right)\left(\psi^{T} i C \gamma_{5} \psi\right)
$$

$$
\left\{\begin{array}{l}
\frac{1}{g}=\int \frac{d^{3} k}{(2 \pi)^{3}}\left[\frac{1-2 f\left(E_{k}^{-}\right)}{2 E_{k}^{-}}+\frac{1-2 f\left(E_{k}^{+}\right)}{2 E_{k}^{-}}\right] \\
n=\int \frac{d^{3} k}{(2 \pi)^{3}}\left[\left(1-\frac{\xi_{k}^{-}}{E_{k}^{-}}\right)\left(1-2 f\left(E_{k}^{-}\right)\right)-\left(1-\frac{\xi_{k}^{+}}{E_{k}^{+}}\right)\left(1-2 f\left(E_{k}^{+}\right)\right)\right]
\end{array}\right.
$$

fermion propagator with diagonal and off-diagonal elements in Nambu-Gorkov space

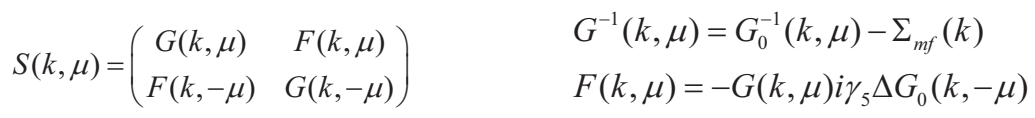

bare propagator and condensate induced self-energy

$$
G_{0}^{-1}(k, \mu)=\left(k_{0}+\mu\right) \gamma_{0}-\vec{\gamma} \cdot \vec{k}-m \quad \Sigma_{m f}(k)=-\Delta^{2} G_{0}(-k, \mu)
$$

gap and number equations in terms of the fermion propagator

$$
\Delta\left[1+i \frac{g}{2} \sum_{k} \operatorname{Tr}\left[G(k, \mu) G_{0}(-k, \mu)\right]=0, \quad n=-i \sum_{k} \operatorname{Tr}\left[\gamma_{0} G(k, \mu)\right]\right.
$$

Matsubara frequency summation $\sum_{k}=i T \sum_{n} \int_{\frac{d^{3} k}{(2 \pi)^{3}},} \quad k_{0} \rightarrow i \omega_{n}, \quad \omega_{n}= \begin{cases}2 n \pi T, & \text { bosons } \\ (2 n+1) \pi T, & \text { fermions }\end{cases}$

July, 2008 Summer School on Dense Matter and HI Dubna 18




\section{mean field theory in $G_{0} G$ scheme}

introducing a condensed-pair propagator

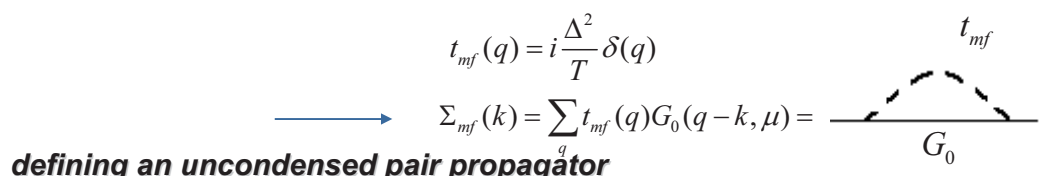

defining an uncondensed pair propagator

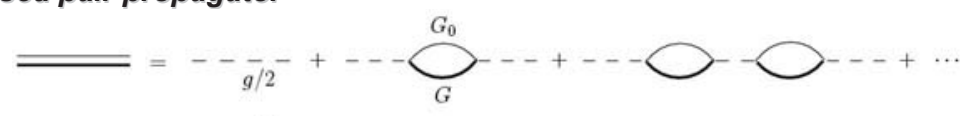

the name $G_{0} G$ scheme,

$G$ is the mean field

fermion propagator

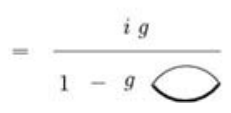

$$
t(q)=\frac{i g}{1-g \chi(q)} \quad \chi(q)=-\frac{i}{2} \sum_{k} \operatorname{Tr}\left[G(k, \mu) G_{0}(q-k, \mu)\right]
$$

gap equation in condensed phase is determined by uncondensed pairs

$$
t^{-1}(0)=0 \longrightarrow \Delta(T, \mu)
$$

problem:

there is no feedback of the uncondensed pairs on the fermion self-energy

July, 2008 Summer School on Dense Matter and HI

Dubna 19

going beyond mean field in $G_{0} G$ scheme

full pair propagator He, Zhuang, 2007

$$
\begin{aligned}
& t(q)=t_{m f}(q)+t_{p g}(q) \\
& t_{m f}(q)=i \frac{\Delta^{2}}{T} \delta(q) \\
& t_{p g}(q)=\frac{i g}{1-g \chi(q)}=
\end{aligned}
$$

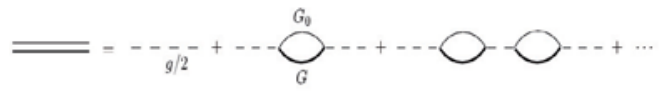

with full susceptibility and full propagator

$$
\chi(q)=-\frac{i}{2} \sum_{k} \operatorname{Tr}\left[G(k, \mu) G_{0}(q-k, \mu)\right]
$$$$
=\frac{i g}{1-9 \bigcirc}
$$$$
G^{-1}(k, \mu)=G_{0}^{-1}(k, \mu)-\Sigma(k)
$$

full fermion self-energy $\Sigma(k)=\sum_{q} t(q) G_{0}(q-k, \mu)$

$$
=\Sigma_{m f}(k)+\Sigma_{p g}(k)=
$$
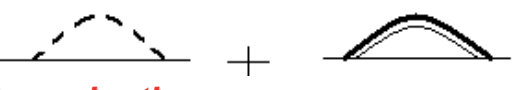

fermions and pairs are coupled to each other

new gap equation $\quad t_{p g}^{-1}(0)=0$ a new order parameter $\Delta(T, \mu)$ which is different from the mean field one

all the formulas look the same as the mean field ones, but we do not know the expression of the full fermion propagator $G$. 
1) $t_{p g}^{-1}(0)=0 \longrightarrow t_{p g}(q)$ peaks at $q=0$

$$
\begin{gathered}
\sum_{p g}(k)=\sum_{q \neq 0} t_{p g}(q) G_{0}(q-k, \mu) \square-\left(-\sum_{q \neq 0} t_{p g}(q)\right) G_{0}(-k, \mu)=-\Delta_{p g}^{2} G_{0}(-k, \mu) \\
\Delta_{p g}^{2}=-\sum_{q \neq 0} t_{p g}(q) \quad \begin{array}{l}
\text { the pseudogap is related to the uncondensed } \\
\text { pairs and does not change the symmetry! }
\end{array}
\end{gathered}
$$

full self-energy $\Sigma(k)=\Sigma_{m f}(k)+\Sigma_{p g}(k) \square-\left(\Delta^{2}+\Delta_{p g}^{2}\right) G_{0}(-k, \mu)$

gap equation $t_{p g}^{-1}(0)=0 \longrightarrow$ mean field gap equation with $\Delta \rightarrow \sqrt{\Delta^{2}+\Delta_{p g}^{2}}$

2) $t_{p g}^{-1}(0)=0 \longrightarrow$ expansion around $q=0$

$$
\begin{aligned}
& t_{p g}(q)=\frac{i g}{1-g \chi(q)}=\frac{-i}{\chi(q)-\chi(0)} \square \frac{-i}{Z_{1} q_{0}+Z_{2} q_{0}^{2}-\xi^{2} \vec{q}^{2}} \\
& Z_{1}=\left.\frac{\partial \chi}{\partial q_{0}}\right|_{q_{0}=0}, \quad Z_{2}=\left.\frac{1}{2} \frac{\partial^{2} \chi}{\partial q_{0}^{2}}\right|_{q_{0}=0}, \quad \xi_{2}=-\left.\frac{1}{2} \frac{\partial^{2} \chi}{\partial \vec{q}^{2}}\right|_{q=0}
\end{aligned}
$$

mean field gap equation with $\Delta \rightarrow \sqrt{\Delta^{2}+\Delta_{p g}^{2}}$

$$
\begin{aligned}
& \Delta_{p g}^{2}=\frac{1}{Z_{2}} \int \frac{d^{3} \vec{q}}{(2 \pi)^{3}} \frac{f_{b}\left(\omega_{q}-v\right)+f_{b}\left(\omega_{q}+v\right)}{2 \omega_{q}} \longrightarrow \Delta(T, \mu), \Delta_{p g}(T, \mu) \\
& v=Z_{1} / 2 Z_{2}, \quad \omega_{q}=\sqrt{\xi^{2} \vec{q}^{2} / Z_{2}+v^{2}}
\end{aligned}
$$

July, 2008 Summer School on Dense Matter and HI Dubna 21

\section{thermodynamics}

$$
\begin{aligned}
& \Omega=\Omega_{m f}+\Omega_{f l} \\
& \Omega_{m f}=\frac{\Delta^{2}}{g}+\int \frac{d^{3} k}{(2 \pi)^{3}}\left[\left(\xi_{k}^{+}+\xi_{k}^{-}-E_{k}^{+}-E_{k}^{-}\right)-\frac{1}{\beta} \ln \left(\left(1+e^{-\beta E_{k}^{-}}\right)\left(1+e^{-\beta E_{k}^{+}}\right)\right)\right] \\
& \Omega_{f l}=\sum_{q} \ln [1-g \chi(q)] \square \frac{1}{\beta} \int \frac{d^{3} q}{(2 \pi)^{3}} \ln \left(\left(1-e^{-\beta \omega_{q}^{-}}\right)\left(1-e^{-\beta \omega_{q}^{+}}\right)\right) \\
& =\left\{\begin{array}{lr}
\frac{1}{\beta} \int \frac{d^{3} q}{(2 \pi)^{3}} \ln \left(1-e^{-\beta q^{2} / 2 m_{B}}\right) & Z_{1}>>Z_{2}, \\
\frac{2}{\beta} \int \frac{d^{3} q}{(2 \pi)^{3}} \ln \left(1-e^{-\beta c q}\right) & Z_{2}>>Z_{1}, \quad \text { relativistic boson gas }
\end{array}\right.
\end{aligned}
$$

\section{number of bosons}

$$
n_{B}=\frac{n}{2}-\int \frac{d^{3} k}{(2 \pi)^{3}}\left[f\left(\xi_{k}^{-}\right)-f\left(\xi_{k}^{+}\right)\right]=Z_{1}\left(\Delta^{2}+\Delta_{p g}^{2}\right)=n_{B}^{m f}+n_{B}^{f l}
$$

\section{fraction of condensed pairs}

$$
P_{c}=\frac{n_{B}^{m f}}{n / 2}=\frac{Z_{1} \Delta^{2}}{n / 2}
$$




\section{BCS-NBEC-RBEC crossover}

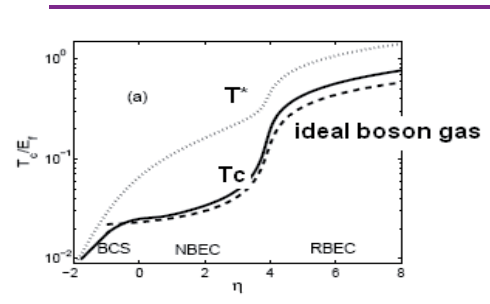

$T_{c}: \quad$ critical temperature

$T<T_{c}: \quad \Delta \neq 0$, condensed phase

$T>T_{c}: \Delta=0$, normal or pseudogap phase

$T^{*}$ : pair dissociation temperature

$T_{c}<T<T^{*}: \Delta_{p g} \neq 0$, pseudogap phase

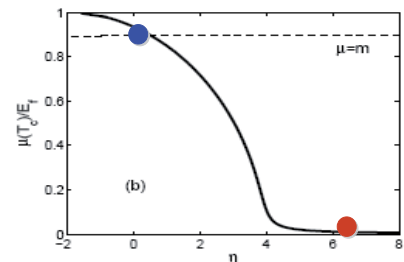

$T>T^{*}: \quad \Delta_{p g}=0 \quad$ normal phase

BCS: $\quad \eta<0, \quad \mu>m$ no pairs

NBEC: $0<\eta<m / k_{F}, \quad 0<\mu<m$ heavy pairs, no anti-pairs RBEC: $\eta>m / k_{F}, \mu \square 0 \quad$ light pairs, almost the same number of pairs and anti-pairs
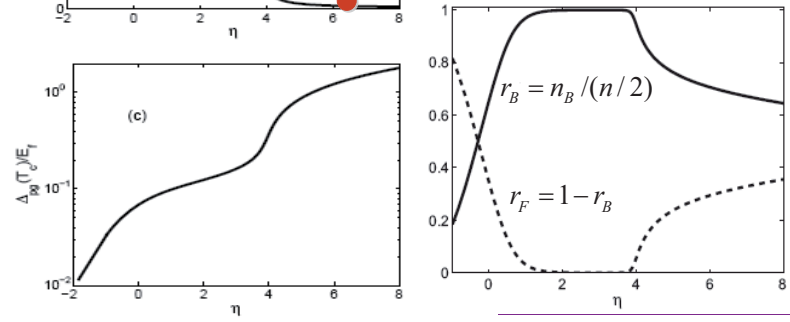

number fractions at $T_{c}$, in RBEC, $T_{c}$ is large enough and there is a strong competition between condensation and dissociation.

July, 2008

discussion on $G_{0} G$

can the symmetry be restored in the pseudogap phase?

fermion propagator including fluctuations (to the order of $\Delta^{2} / \Lambda^{2}$ ) :

$$
S^{-1}(k)=\left(\begin{array}{cc}
G_{0}^{-1}(k, \mu)-\Sigma_{p g}(k, \mu) & i \gamma_{5} \Delta \\
i \gamma_{5} \Delta & G_{0}^{-1}(k, \mu)-\Sigma_{p g}(k,-\mu)
\end{array}\right)
$$

the pseudogap appears in the diagonal elements of the propagator and does not break the symmetry of the system.

\section{Kandanoff and Martin:}

the scheme $\chi \square G G$ can not give a correct symmetry restoration picture and the specific heat $C_{V} \square T^{2}$ is wrong. 


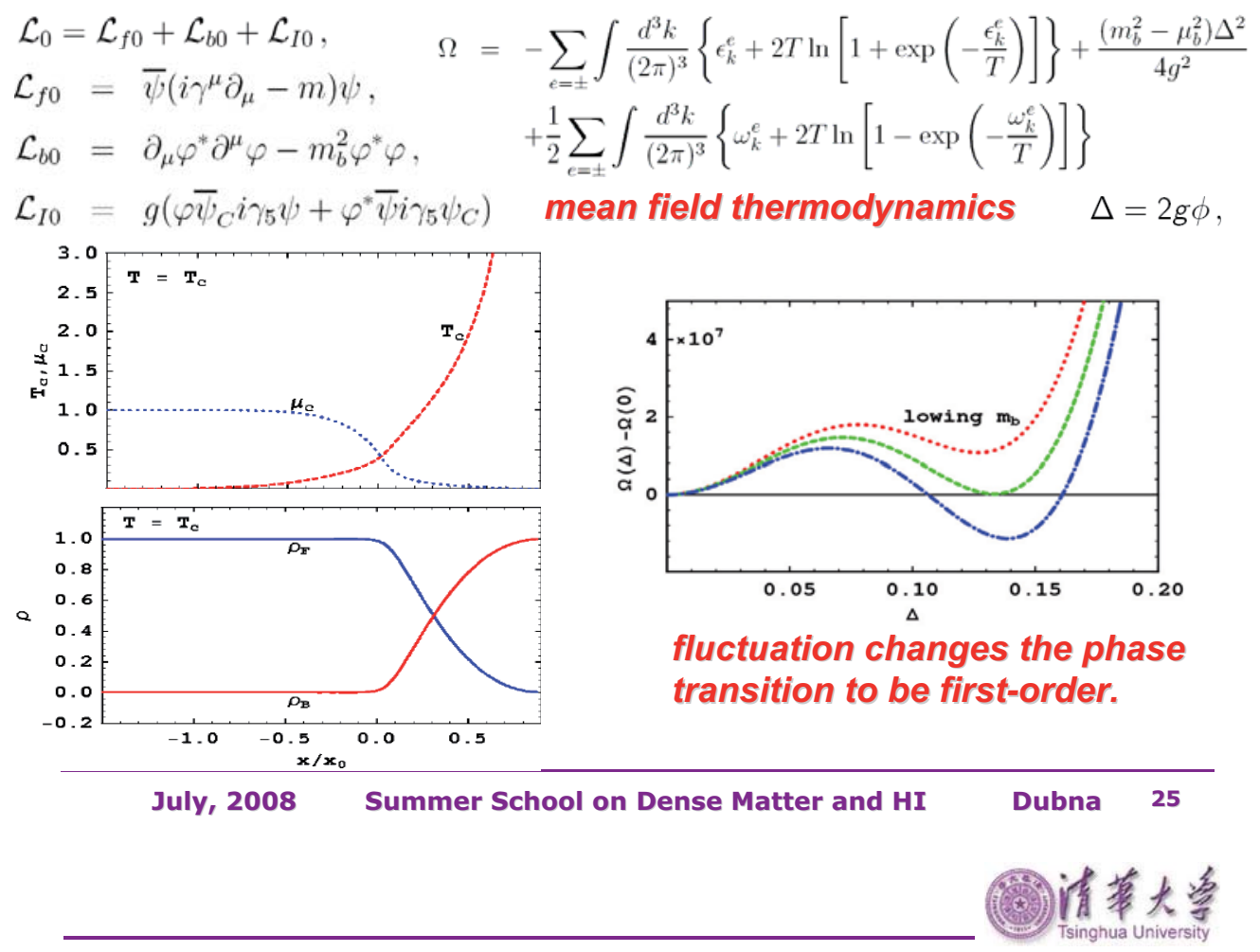

4) Application to QCD: Color Superconductivity and Pion Superfluid 
* QCD phase transitions like chiral symmetry restoration, color superconductivity, and pion superfluid happen in non-perturbative temperature and density region, the coupling is strong.

* relativistic BCS-BEC crossover is controlled by $\mu-m$, the BEC-BCS crossover would happen when the light quark mass changes in the QCD medium.

* effective models at hadron level can only describe BEC state, they can not describe BEC-BCS crossover. One of the models that enables us to describe both quarks and mesons and diquarks is the NJL model at quark level.

$L_{N L}=\bar{\psi}\left(i \gamma^{\mu} \partial_{\mu}-m_{0}+\mu \gamma_{0}\right)+G_{S}\left((\bar{\psi} \psi)^{2}+\left(\bar{\psi} i \tau_{i} \gamma_{5} \psi\right)^{2}\right)+G_{D}\left(\bar{\psi}_{i \alpha}^{C} \varepsilon^{i j} \varepsilon^{\alpha \beta \gamma} i \gamma_{5} \psi_{j \beta}\right)\left(\bar{\psi}_{i \alpha} \varepsilon^{i j} \varepsilon^{\alpha \beta \gamma} i \gamma_{5} \psi_{j \beta}^{C}\right)$

\section{disadvantage: no confinement}

* there is no problem to do lattice simulation for real QCD at finite isospin density

July, 2008 Summer School on Dense Matter and HI Dubna 27

\section{color superconductivity}

order parameters of spontaneous chiral and color symmetry breaking

$$
\begin{aligned}
& \sigma=\langle\bar{\psi} \psi\rangle \\
& \Delta=\Delta^{3}=\left\langle\bar{\psi}_{i \alpha}^{C} \varepsilon^{i j} \varepsilon^{\alpha \beta 3} i \gamma_{5} \psi_{j \beta}\right\rangle \text { color breaking from SU(3) to SU(2) }
\end{aligned}
$$

leading order of $1 / \mathrm{N}_{c}$ for quarks, and next to leading order for mesons \& diquarks quark propagator in 12D Nambu-Gorkov space

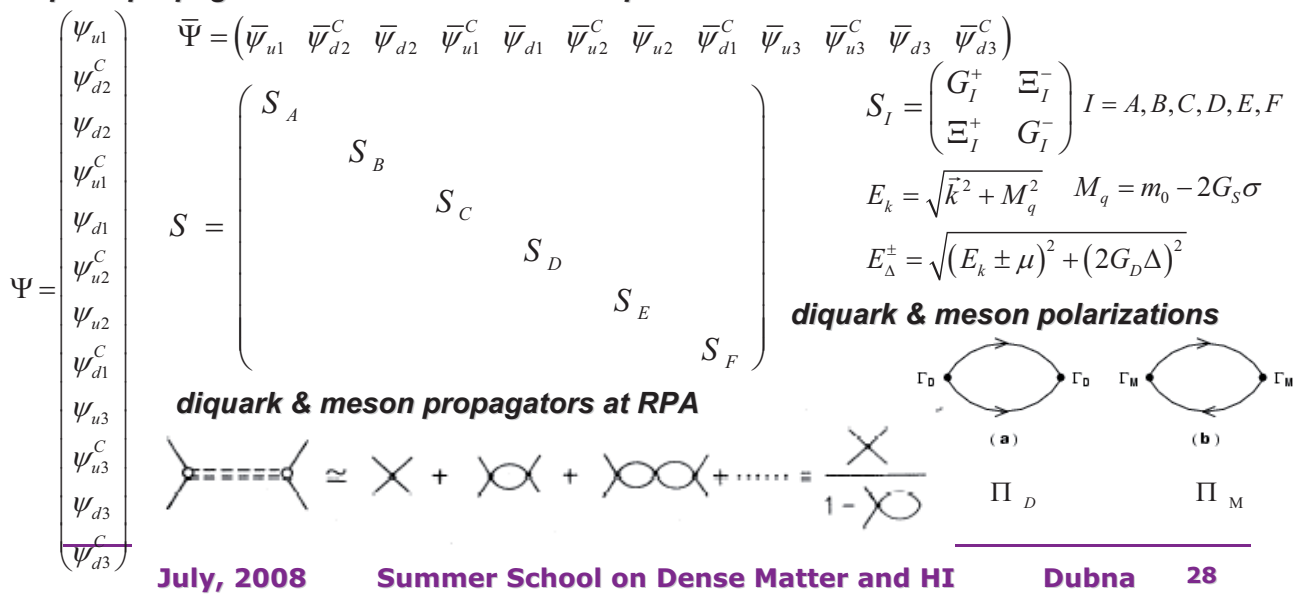


gap equations for chiral and diquark condensates at $T=0$

$$
\left\{\begin{array}{l}
m-m_{0}=8 G_{s} m \int \frac{d^{3} k}{(2 \pi)^{3}} \frac{1}{E_{p}}\left[\frac{E_{k}-\mu_{B} / 3}{E_{\Delta}^{-}}+\frac{E_{k}+\mu_{B} / 3}{E_{\Delta}^{+}}+\Theta\left(E_{k}-\mu_{B} / 3\right)\right] \\
\Delta=8 G_{d} \Delta \int \frac{d^{3} k}{(2 \pi)^{3}}\left[\frac{1}{E_{\Delta}^{-}}+\frac{1}{E_{\Delta}^{+}}\right] \quad \begin{array}{l}
\text { to guarantee color neutrality, we introduce } \\
\text { color chemical potential: }
\end{array}
\end{array}\right.
$$

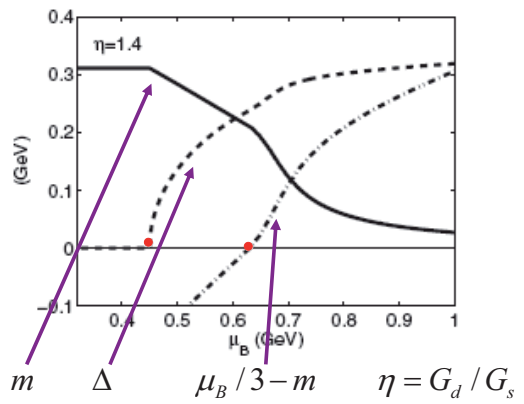

there exists a BCS-BEC crossover

$$
\mu_{r}=\mu_{g}=\mu_{B} / 3+\mu_{8} / 3, \quad \mu_{b}=\mu_{B} / 3-2 \mu_{8} / 3
$$

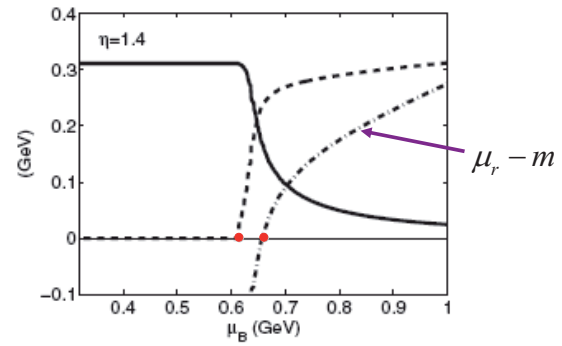

color neutrality speeds up the chiral restoration and reduces the BEC region

July, $2008 \quad$ Summer School on Dense Matter and HI Dubna 29

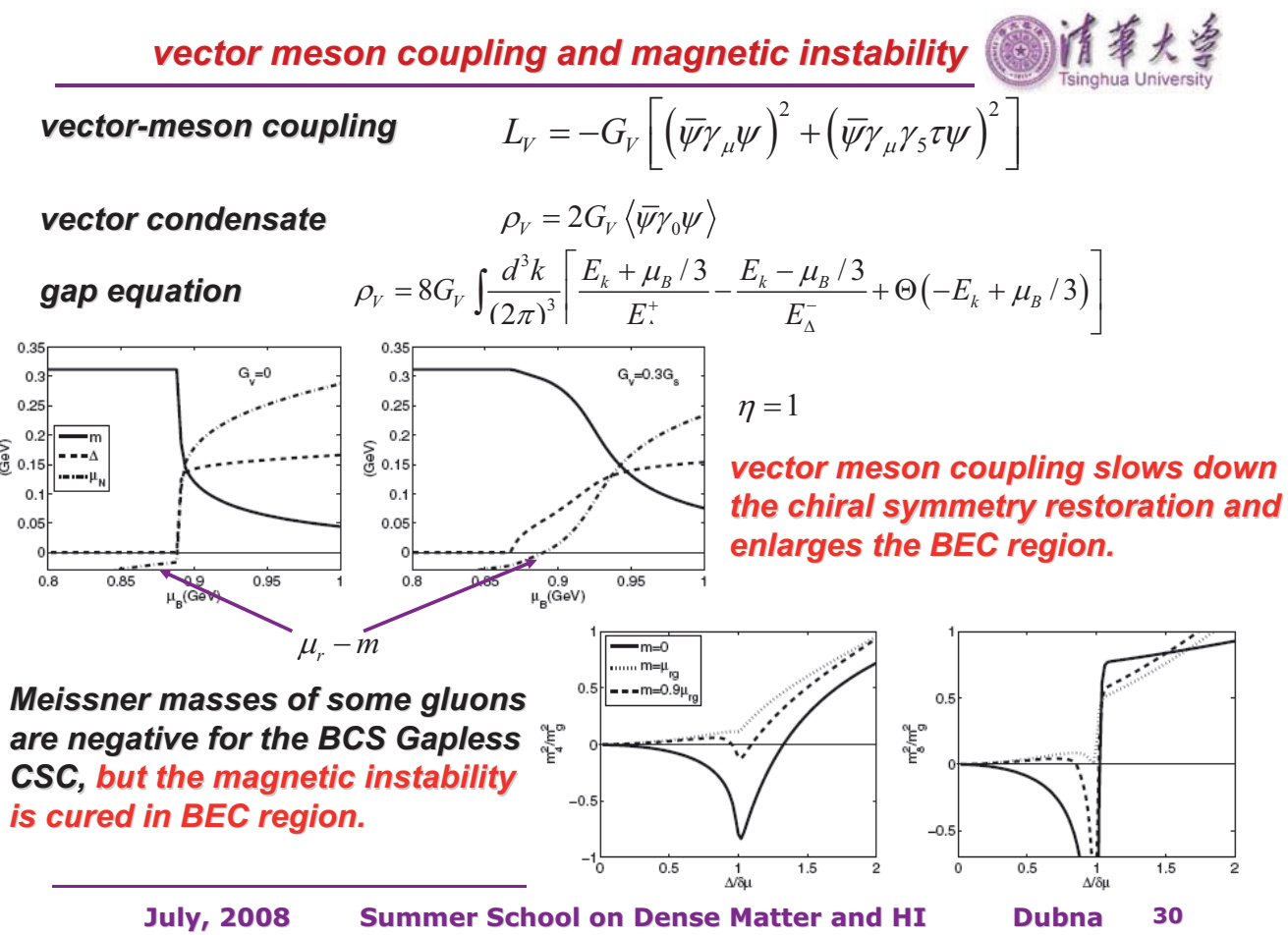




\section{beyond men field}
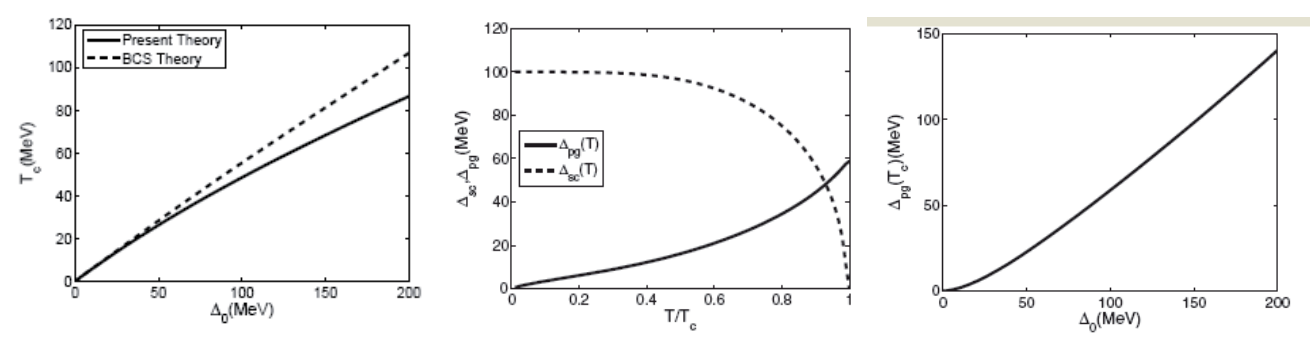

$\Delta_{0}=\Delta(T=0)$ is determined by the coupling and chemical potential

$\Delta_{0}=100-200 \mathrm{MeV} \leftrightarrow \mu_{q}=300-500 \mathrm{MeV}$

- going beyond mean field reduces the critical temperature of color superconductivity pairing effect is important around the critical temperature and dominates the symmetry restored phase

\section{pion superfluid}

\section{NJL with isospin symmetry breaking}

quark chemical potentials

$$
L_{N J L}=\bar{\psi}\left(i \gamma^{\mu} \partial_{\mu}-m_{0}+\mu \gamma_{0}\right) \psi+G\left((\bar{\psi} \psi)^{2}+\left(\bar{\psi} i \tau_{i} \gamma_{5} \psi\right)^{2}\right)
$$

$$
\mu=\left(\begin{array}{cc}
\mu_{u} & 0 \\
0 & \mu_{d}
\end{array}\right)=\left(\begin{array}{cc}
\mu_{B} / 3+\mu_{I} / 2 & 0 \\
0 & \mu_{B} / 3-\mu_{I} / 2
\end{array}\right)
$$

chiral and pion condensates with finite pair momentum

quark propagator in $\mathrm{MF}$

$$
\begin{aligned}
& \sigma=\langle\bar{\psi} \psi\rangle=\sigma_{u}+\sigma_{d}, \quad \sigma_{u}=\langle\bar{u} u\rangle, \quad \sigma_{d}=\langle\bar{d} d\rangle \\
& \pi_{+}=\sqrt{2}\left\langle\bar{u} i \gamma_{5} d\right\rangle=\frac{\pi}{\sqrt{2}} e^{2 i \vec{q} \cdot \vec{x}}, \quad \pi_{-}=\sqrt{2}\left\langle\bar{d} i \gamma_{5} u\right\rangle=\frac{\pi}{\sqrt{2}} e^{-2 i \vec{q} \cdot \vec{x}}
\end{aligned}
$$

$$
S^{-1}(p, \vec{q})=\left(\begin{array}{cc}
\gamma^{\mu} p_{\mu}-\vec{\gamma} \cdot \vec{q}+\mu_{u} \gamma_{0}-m & 2 i G \pi \gamma_{5} \\
2 i G \pi \gamma_{5} & \gamma^{\mu} k_{\mu}+\vec{\gamma} \cdot \vec{q}+\mu_{d} \gamma_{0}-m
\end{array}\right) \quad m=m_{0}-2 G \sigma
$$

\section{thermodynamic potential and gap equations:}

$$
\begin{aligned}
& \Omega=G\left(\sigma^{2}+\pi^{2}\right)-\frac{T}{V} \operatorname{Tr} \operatorname{Ln} S^{-1} \\
& \frac{\partial \Omega}{\partial \sigma_{u}}=0, \quad \frac{\partial^{2} \Omega}{\partial \sigma_{u}^{2}} \geq 0, \quad \frac{\partial \Omega}{\partial \sigma_{d}}=0, \quad \frac{\partial^{2} \Omega}{\partial \sigma_{d}^{2}} \geq 0, \quad \frac{\partial \Omega}{\partial \pi}=0, \quad \frac{\partial^{2} \Omega}{\partial \pi^{2}} \geq 0, \quad \frac{\partial \Omega}{\partial q}=0, \frac{\partial^{2} \Omega}{\partial q^{2}} \geq 0
\end{aligned}
$$


meson propagator $D$ at RPA

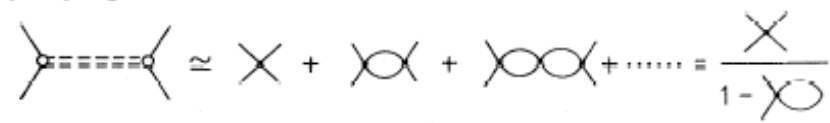

considering all possible channels in the bubble summation

meson polarization functions

$$
\Pi_{m n}(k)=i \int \frac{d^{4} p}{(2 \pi)^{4}} \operatorname{Tr}\left(\Gamma_{m}^{*} S(p+k) \Gamma_{n} S(p)\right) \quad \Gamma_{m}=\left\{\begin{array}{l}
1, \quad m=\sigma \\
i \tau_{+} \gamma_{5}, m=\pi_{+} \\
i \tau_{-} \gamma_{5}, m=\pi_{-} \\
i \tau_{3} \gamma_{5}, m=\pi_{0}
\end{array}\right.
$$

pole of the propagator determines meson masses $M_{m}$

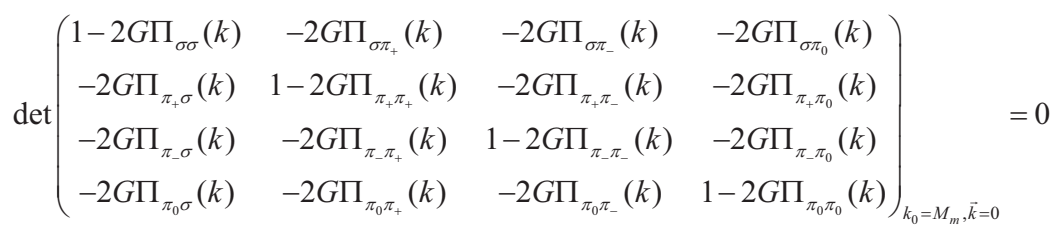

mixing among normal $\sigma, \pi_{+}, \pi_{-}$in pion superfluid phase, the new eigen modes $\bar{\sigma}, \bar{\pi}_{+}, \bar{\pi}_{-}$are linear combinations of $\sigma, \pi_{+}, \pi_{-}$

\section{phase diagram of pion superfluid}
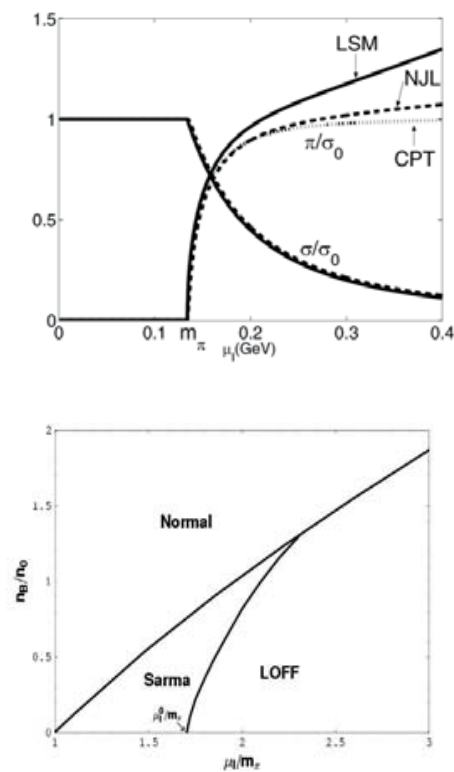

chiral and pion condensates at $T=\mu_{B}=\vec{q}=0$ in NJL, Linear Sigma Model and Chiral Perturbation Theory, there is no remarkable difference around the critical point.

analytic result: critical isospin chemical potential for pion superfluidity is exactly the pion mass in the vacuum:

$$
\mu_{I}^{c}=m_{\pi}
$$

pion superfluidity phase diagram in $\mu_{I}-\mu_{B}$ plane at $T=0$

$\mu_{I}$ : average Fermi surface

$\mu_{B}\left(n_{B}\right):$ Fermi surface mismatch

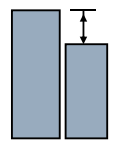

homogeneous (Sarma, $\vec{q}=0$ ) and inhomogeneous pion superfluid (LOFF, $\vec{q} \neq 0$ )

magnetic instability of Sarma state at high average Fermi surface leads to the LOFF state 
BCS-BEC crossover of pion superfluid
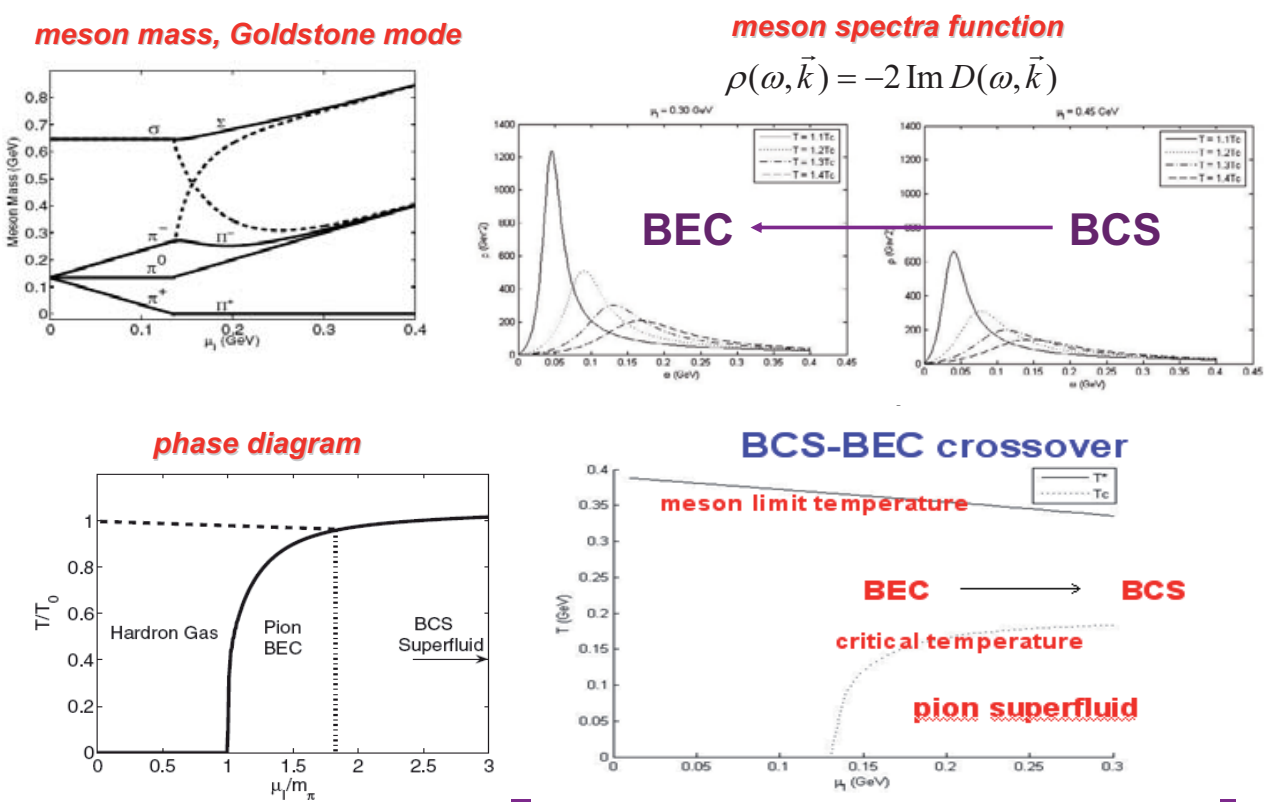

July, 2008

Summer School on Dense Matter and HI

Dubna 35

BCS-BEC crossover in asymmetric nuclear matter

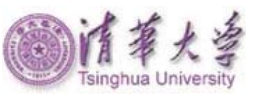

$\star$ transition from BCS pairing to BEC in low-density asymmetric nuclear matter,

U. Lombardo, P. Nozières:PRC64, 064314 (2001)

ઐ spatial structure of neutron Cooper pair in low density uniform matter,

Masayuki Matsuo:PRC73, 044309 (2006)

$\star B C S-B E C$ crossover of neutron pairs in symmetric and asymmetric nuclear matters, J. Margueron: arXiv:0710.4241

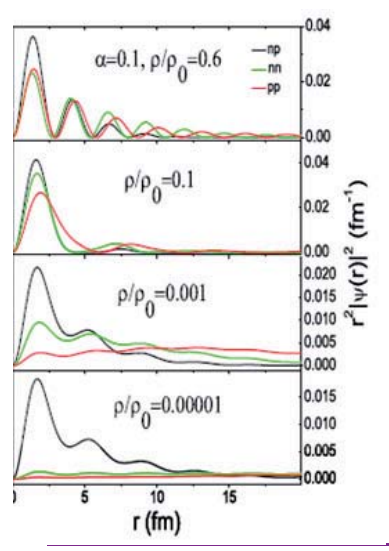

July, 2008 asymmetric nuclear matter with both

np and nn and pp pairings Mao, Huang, Zhuang, 2008

considering density dependent Paris potential and nucleon mass

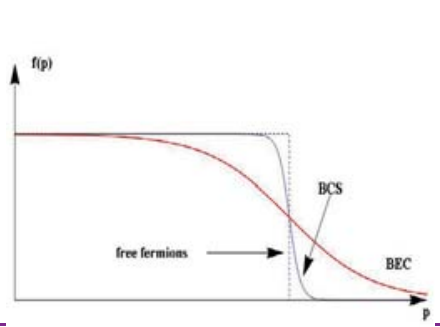

$\psi(\vec{r})=\int \frac{d^{3} p}{(2 \pi)^{3}} e^{i \vec{p} \cdot \vec{r}} \varphi(\vec{p})$

there exists a strong Friedel oscillation in BCS region, and it is washed away in $B E C$ region. 


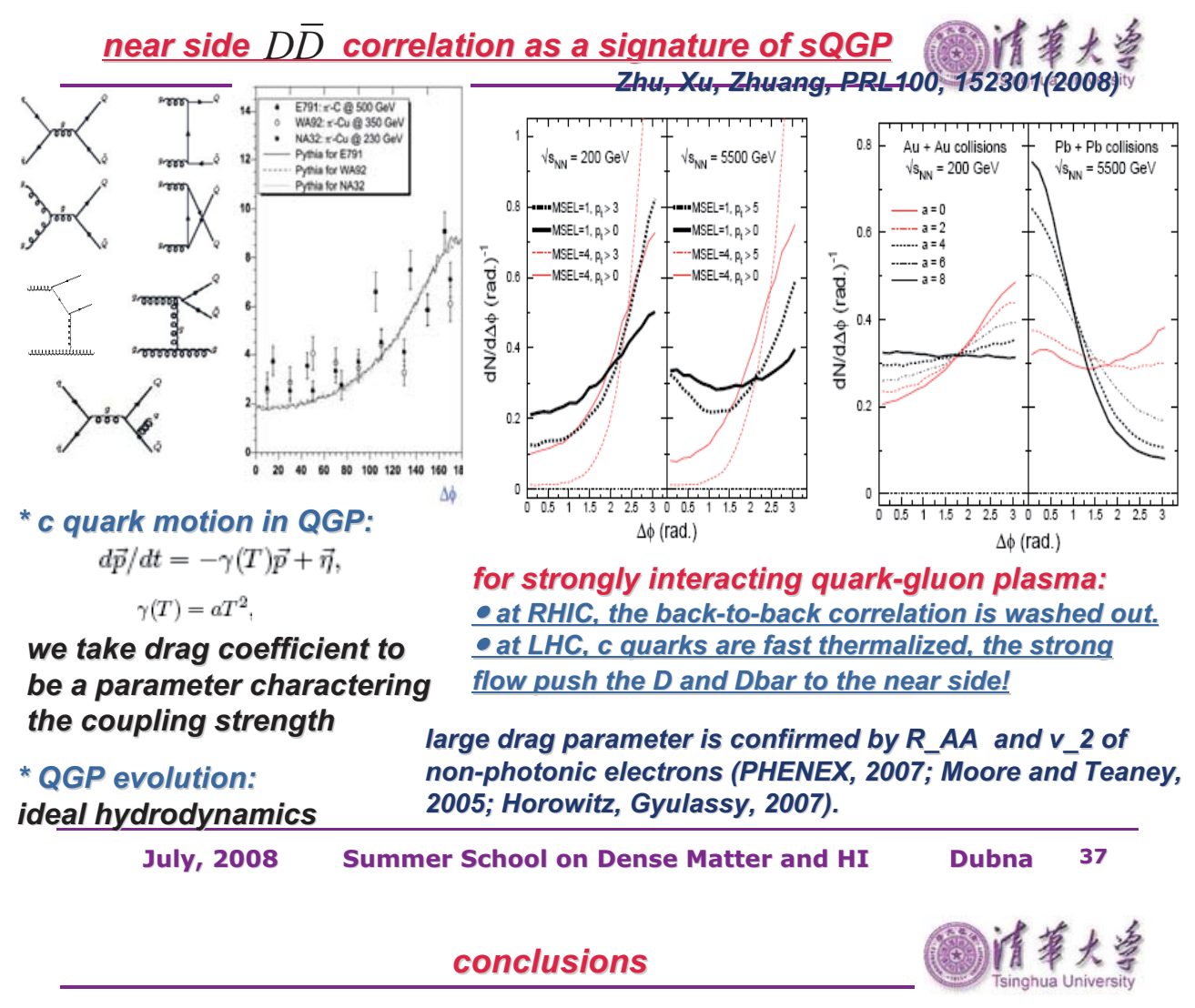

* BCS-BEC crossover is a general phenomena from cold atom gas to quark matter.

* BCS-BEC crossover is closely related to QCD key problems: vacuum, color symmetry, chiral symmetry, isospin symmetry .......

* BCS-BEC crossover of color superconductivity and pion superfluid is not induced by simply increasing the coupling constant of the attractive interaction but by changing the corresponding charge number.

* There are potential applications in heavy ion collisions (at CSR/Lanzhou, FAIR/GSI and RHIC/BNL) and compact stars.

\section{thanks for your patience}




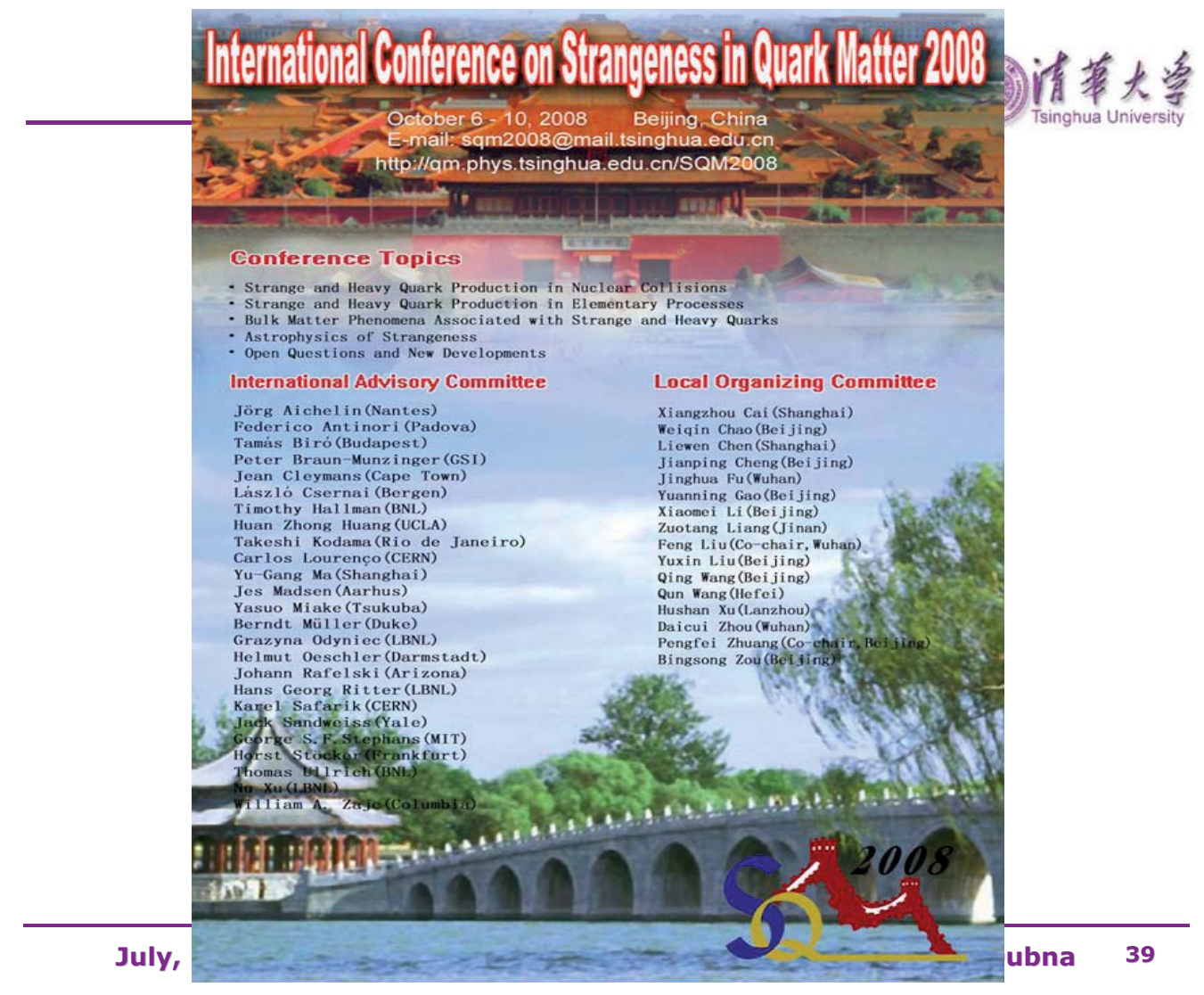

